\title{
CONNECTING THE REAL WORLD AND VIRTUAL WORLD THROUGH GAMING
}

\author{
Adrian David Cheok, Goh Kok Hwee, Liu Wei, Jason Teo, Teo Sze Lee, \\ Farzam Farbiz, Lee Shang Ping \\ Mixed Reality Lab, National University of Singapore
}

\begin{abstract}
This paper presents two novel interactive game entertainment systems named by Human Pacman and Touch spaced that ventures to embed the natural physical world seamlessly with a fantasy virtual playground by capitalizing on mobile computing, wireless LAN, ubiquitous computing, and motion-tracking technologies. We can connect seamlessly the computer virtual world to our real world through these game systems. Human Pacman and Touch space are physical roleplaying augmented-reality computer fantasy together with real human-social and mobile gaming. They recapture human touch and physical interaction real-world environment as essential elements of the game play, whilst also maintaining the exciting fantasy features of traditional computer entertainment. It emphasizes collaboration and competition between players in a wide indoor and outdoor physical area which allows natural wide-area human-physical movements.
\end{abstract}

Keywords: Collaboration, Physical interaction, Social computing, Wearable computing, Tangible interaction, Ubiquitous computing

\section{Introduction}

In the pre-computer age, games were designed and played out in the physical world with the use of real-world properties, such as physical objects, our sense of space, and spatial relations. Nowadays, computer games focus the user's attention mainly on the computer screen or a 2-D/3-D virtual environment, thereby, constraining physical interactions. However, there seems to be a growing interest in physical gaming and entertainment, even in industry. Commercial arcade games have recently seen a growing trend of games that require human-physical movement as part of interaction. For example, dancing games such as Dance Dance Revolution and ParaParaParadise are based on players dancing in time with a musical dance tune and moving graphical objects. However, these systems still force the person to stand in more or less 
the same spot, and focus on a computer screen in front of them. Nevertheless, the underpinning philosophy is similar.

In this paper we propose two novel game systems that we developed in our laboratory which bring both features of physical gaming and computer gaming together. In a better word, our systems connect the real world and physical interaction between players to the computer world (virtual world) through the game space. The players can have social interaction with each others and physical movements like traditional games and at the same time they will enjoy interacting with virtual objects (e.g. monsters, witches, ...) in both real and virtual environment. All the players' movements in real world are tracked by the game system to update their virtual world in real time.

The organization of the paper is as follows. We illustrate our game system, Human Pacman, in Section 2 and then in Section 3 we give the explanation of the second system, Touch Space. In Section 4 we provide the conclusion.

\section{Human Pacman}

In recent years, the world has seen the proliferation of highly portable devices, such as personal digital assistants (PDAs), laptops, and cellular telephones. Trends in computing environment development also suggest that users are gradually being freed from the constraints of stationary desktop computing with the explosive expansion in mobile computing and networking infrastructure. With this technological progress in mind, we have developed human Pacman , a genre of computer entertainment that is based on real-world-physical, social, and wide-area mobile-interactive entertainment. The novelty of this computer game has the following aspects: first, the players physically and immersively role-play the characters of the Pacman and the Ghosts, as if a fantasy computer digital world has merged with the real physical world. Second, users can move about freely in the real world over wide area indoor and outdoor spaces whilst maintaining seamless networked social contact with human players in both the real and virtual world. Third, human Pacman also explores novel tangible aspects of human physical movement and perception, both in the player's environment and in the interaction with the digital world. In other words, objects in the real world are embedded and take on a real-time link and meaning with objects in the virtual world. For example, to devour the virtual "enemy", the player has to tap on the real physical enemy's shoulder; to obtain a virtual "magic" cookie, the player has to physically pick up a real physical treasure box with an embedded Bluetooth device attached. Human Pacman ventures to elevate the sense of thrill and suspended disbelief of the players in this untypical computer game. . Each of the novel interactions mentioned is summarized in Table 1. 
Human Pacman features a centralized client-server architecture that is made up of four main entities, namely, a central server, client wearable computers, helper laptops, and Bluetooth-embedded objects. Wireless LAN serves as a communication highway between the wearable computers, the helper computers (laptops), and the server desktop computer.

In this game the players are assigned to two opposing teams, namely the Pacman team and the Ghost team. The former consists of two Pacmen and two Helpers; correspondingly, the latter consists of two Ghosts and two Helpers. Each Pacman or Ghost is in coalition with one Helper, promoting collaboration and interaction between the users. Since a Helper player is essentially participating in the game play remotely by using a computer terminal over a wireless LAN, human Pacman can effectively be expanded to include online players anywhere on Earth who can view and collaborate, via the Internet, with real human Pacmen and Ghosts who are immersed in the physical playground.

Ever since its introduction by Namco to Japanese arcade fans in 1979, Pacman has gone through numerous stages of development. Yet, the ultimate goal of the game remains fundamentally unchanged. We have designed human Pacman to be in close resemblance to the original Pacman in terms of game objectives so that the players' learning curves are very much leveled to the point where they can pick up the game in very little time and enjoy the associated familiarity. Basically, the goal of the Pacman team is to collect all virtual plain cookies and hidden special cookies in Pac-World whilst avoiding the Ghosts. On the other hand, the aim of the Ghost team is to devour all Pacmen in PacWorld. To add to the excitement of the game play, after "eating" a special cookie, a Pacman gains Ghost-devouring capability and, henceforth, can attack her enemy for a limited period of time.

Pac-World is a fantasy world existing simultaneously in physical reality, in AR and VR modes. Pacmen and Ghosts, who are walking around in the real world with their networked wearable computers and HMD, view the world in AR mode. Helpers, on the other hand, can view it in VR mode since they are stationed in front of networked computers. Most importantly, there is a direct and real-time link between the wide-area physical world and the virtual PacWorld at all times, thus, providing the users with a ubiquitous and seamless merging of the fantasy digital world and the realistic physical world. Here we have converted the real world to a fantasy virtual playground by ingraining the latter with direct physical correspondences.

The real-time position of each mobile user is sent periodically to the server through the wireless LAN. Upon receiving the position data, the server sends an update to each wearable computer detailing the position of the other mobile players, as well as the positions of all "non-eaten" plain cookies.

Pacman has to physically move within the game area to collect all virtual plain cookies overlaid in the real world, by walking through them as seen 
through her HMD. Such a physical action is reflected visually in Pac-World through the disappearance of the cookie in both the AR and VR mode. When she walks through the cookie, the cookie disappears. This collection is also reflected in real time in the virtual Pac-World (seen by the Helpers) and the Pac-World map (seen by both Pacmen and Ghosts) through the disappearance of the cookie in the corresponding location.

In addition, she has to find and collect special cookies in the virtual PacWorld. These are directly linked and represented by Bluetooth-embedded objects. This creates a sense of presence and immersion within the virtual PacWorld, as well as a feeling of active participation in the real world. Pacman collects a special cookie by touching real Bluetooth-embedded objects placed in different parts of the game area. When the Pacman is within range of the Bluetooth object (a distance of about $10 \mathrm{~m}$ ), communication takes place between the wearable computer and the Bluetooth device.

The collection of the special cookie exemplifies a natural tangible interaction involving physically interacting with this object through human touch. Pacman is able to hold a real object naturally in her hands as should be in reallife treasure finding. Such a tangible action provides the player with a sense of touch in the fantasy domain of the game play. The Ghost can devour a Pacman by tapping on a capacitive sensor attached to a Pacman's shoulder. Likewise, a Ghost can be devoured by Pacmen endowed with Ghost-devouring powers. Such tangible physical interaction between humans, commonly found in traditional games such as hide-and-seek and the classic "catching" game, is now revived in this computer gaming arena.

Each Pacman and Ghost will be assigned a partner Helper who acts as an intelligence, advisor, and coordinator in her quest to achieve her goal. To enhance the gaming experience for both Pacmen and Ghosts, these players in the physical world with wearable computers are not able to see enemy mobile units (the positions of the enemies are not shown on the virtual map, and there is no AR labeling on them) and hidden special cookies. The Helper, who is in VR mode and sees all, guides her partner by messaging her with important information. This promotes collaboration and interaction between players through the internet.

\section{Touch Space}

In Touch-Space, the games are situated and carried out in the physical world, and they recognize the physical co-location of players and objects in the world as essential elements of the game mechanics. Players must walk around within a large room-size area and pick up real objects to physically interact with the game space, in the similar way as they are playing a traditional non-computer game. What enhances the physical space is that the real object and real envi- 
Table 1. Detailed descriptions of the features of human Pacman

\begin{tabular}{|c|c|}
\hline Feature & Details \\
\hline Physical gaming & $\begin{array}{l}\text { Players are physically role-playing the characters of Pacman and } \\
\text { Ghosts; with wearable computers donned, they use free bodily } \\
\text { movements as part of interaction between each person, between } \\
\text { the real and virtual world, and among objects in the real wide-area } \\
\text { landscapes and virtual environments }\end{array}$ \\
\hline Social gaming & $\begin{array}{l}\text { Players interact both directly with other players when they are in } \\
\text { physical proximity, or indirectly via the wireless LAN network by } \\
\text { real-time messaging. There is a coherent networked social contact } \\
\text { among }\end{array}$ \\
\hline Mobile gaming & $\begin{array}{l}\text { players in both the real and virtual worlds, as well as throughout } \\
\text { their boundaries. People from all around the world can also partici- } \\
\text { pate in the human Pacman experience by viewing and collaborating } \\
\text { in real time over the internet with the physical human Pacmen and } \\
\text { Ghosts who are immersed in the physical-real-world game Players } \\
\text { are free to move about in the indoor/outdoor space without being } \\
\text { constrained to the 2D/3D screen of desktop computers }\end{array}$ \\
\hline $\begin{array}{l}\text { Ubiquitous com- } \\
\text { puting }\end{array}$ & $\begin{array}{l}\text { Everyday objects throughout the environment seamlessly have a } \\
\text { real-time fantasy digital world link and meaning. There is auto- } \\
\text { matic communication between wearable computers and Bluetooth } \\
\text { devices embedded in physical objects used in game play }\end{array}$ \\
\hline $\begin{array}{l}\text { Tangible interac- } \\
\text { tion }\end{array}$ & $\begin{array}{l}\text { Throughout the game, people interact in a graspable and tangible } \\
\text { manner. For example, players need to physically pick up objects to } \\
\text { collect them digitally, or to tap on the shoulder of other players to } \\
\text { devour them }\end{array}$ \\
\hline $\begin{array}{l}\text { Outdoor wide-area } \\
\text { gaming arena }\end{array}$ & $\begin{array}{l}\text { Large outdoor areas can be set up for the game whereby players } \\
\text { carry out their respective missions for the role they play. This could } \\
\text { even be linked throughout cities }\end{array}$ \\
\hline
\end{tabular}

ronment may be augmented with virtual objects or virtual figures. Thus, the benefits and excitement of computer entertainment is also incorporated into the physical space. The system supports multiple simultaneous participants playing together, while maintaining the social, non-mediated interaction between players. Through a seamless traversable interface, the players can transit to and from fully immersive virtual environment. Thus, players will experience a novel full spectrum game experience ranging from physical reality, augmented reality, to virtual reality, in a seamless way featured with tangible interfaces and social interaction.

The story of the game is as follows: a princess is captured by a witch and is trapped in the witch's castle that is located in a mysterious land. To play the game, firstly the two players need to find two map pieces of the mysterious land and other necessary treasures. Secondly, they should fly above the land 
and look for the castle, after then, they need to fight and defeat the witch. Finally, they enter the castle to find the princess, and thus they complete the game mission. Thus, the game consists of three main game stages stages.

Touch-Space is an exploration of the embodied interaction within a mixed reality collaborative setting. The result of the project is a unique game space which combines the interactions of natural human to human, human to physical world and human to virtual world, and provides a novel game experience ranging from physical reality, augmented reality, to virtual reality.

\section{Conclusion}

The continual propagation of digital communication and entertainment in recent years has forced many changes in societal psyche and lifestyle, that is, how we think, work, and play. With physical and mobile gaming gaining popularity, traditional paradigms of entertainment will irrevocably shake one from the stale television set inertia. We believe that human Pacman and Touch Space herald the conjuration and growth of a new genre of computer game that are built on mobility, physical actions, and the real world as a playground. Reality, in this case, is becoming more exotic than fantasy because of the mixed reality element in the game play. On the other hand, emphasis on physical actions might even bring forth the evolvement of professional physical gaming as a competitive sport of the future, for example "Pacman International League". Furthermore, by providing seamless transition between physical world and virtual world, the players can enjoy both tangible physical game experience and fantasy virtual game experience and, therefore, a higher than ever level of sensory gratification is obtained. These games connect the computer generated virtual world to our real world to each other.

We envision a new type of game experience that has two main features: integrated ubiquitous context-awareness and sociality into the computer interaction context, which entails ubiquitous, tangible, and social computing (and thus directly applies the theory of embodied interaction); and a seamless merging of physical world, augmented world and virtual world exploration experience. 